\title{
De la banalité de la violence à la terreur : le cas colombien
}

Daniel Pécaut

\section{OpenEdition}

1 Journals

Édition électronique

URL : http://journals.openedition.org/conflits/2169

DOI : 10.4000/conflits.2169

ISSN : $1777-5345$

Éditeur :

CCLS - Centre d'études sur les conflits lilberté et sécurité, L'Harmattan

Édition imprimée

Date de publication : 15 mai 1997

ISSN : 1157-996X

Référence électronique

Daniel Pécaut, « De la banalité de la violence à la terreur : le cas colombien », Cultures \& Conflits [En ligne], 24-25 | hiver 1996- printemps 1997, mis en ligne le 27 mars 2007, consulté le 30 mars 2021. URL : http://journals.openedition.org/conflits/2169; DOI : https://doi.org/10.4000/conflits.2169

Ce document a été généré automatiquement le 30 mars 2021.

Creative Commons License 


\title{
De la banalité de la violence à la terreur : le cas colombien
}

\author{
Daniel Pécaut
}

1 Depuis 1980, la Colombie est à nouveau le théâtre d'une violence d'une exceptionnelle intensité. Le taux national d'homicides y dépasse régulièrement 70 pour 100.000 habitants, un des taux les plus élevés au monde. Dans certaines régions ou villes du pays, le taux atteint 400 pour 100.000. De 1980 à 1995, le bilan des victimes s'élève à plus de $300.000^{1}$, Les massacres collectifs de cinq personnes ou plus sont innombrables. Pour les seules années 1988-1993, on en compte près de 900 qui ont fait plus de 5.000 victimes ${ }^{2}$. Bien d'autres indicateurs vont dans le même sens. Les militants politiques et syndicaux assassinés se comptent par milliers. Un parti politique, l'Union Patriotique (UP), a été décimé ${ }^{3}$ et pratiquement rayé de la carte. Le nombre des enlèvements officiellement recensés oscille depuis 1990 entre 1000 et 1717, record atteint en 1991. Plus de 500.000 personnes ont dû abandonner leur région d'origine. Les pratiques de racket et de chantage, sous les formes les plus diverses, sont devenues routinières sur une grande partie du territoire.

2 Semblable violence se traduit dans maintes zones rurales et urbaines par des situations de terreur. C'est notamment le cas là où plusieurs forces se disputent un même territoire, le Moyen Magdalena et la région d'Urabá par exemple. Massacres, exodes, exactions, atrocités, peur, suspicion ne cessent d'y être à l'ordre du jour, affectant une population civile soumise à la loi du silence. Les années 1987-1993 ont été de surcroît marquées par le terrorisme, aveugle ou ciblé, mis en œuvre par les narcotrafiquants et les forces obscures alliées avec eux.

3 L'on ne peut donc que s'étonner qu'un tel état de choses ne donne pas lieu à plus de réactions de l'opinion colombienne ou internationale. Certains événements ont eu sans doute un retentissement considérable, tels les assassinats de dirigeants politiques de premier plan ou certains massacres particulièrement meurtriers. Mais ce retentissement n'est que fugace et l'on ne voit pas que la situation d'ensemble donne lieu à une indignation comparable à celle qu'ont suscitée les atrocités en Argentine, au Guatemala ou au Salvador. 
Divers facteurs peuvent expliquer ce silence relatif. Je mettrai l'accent dans cette étude sur l'un d'entre eux : la banalité de la violence, qui entraine la faible visibilité des situations de terreur. En parlant de banalité de la violence, je n'entends pas reprendre à mon compte l'idée d'une " culture de la violence " qui revient souvent sous la plume d'analystes colombiens. Comme souvent, l'explication par la culture, et plus encore s'agissant de la violence, risque d'être quelque peu paresseuse et de revêtir un aspect tautologique. Il est bien vrai que les phénomènes de violence ont une longue histoire en Colombie et que la mémoire de l'épisode de la Violencia de 1950 reste présente dans tous les esprits et est invoquée comme justification par nombre de protagonistes de la violence actuelle. On ne saurait néanmoins ignorer les composantes nouvelles de la violence actuelle. La banalité tient à plusieurs facteurs propres aux phénomènes présents : leurs dimensions extrêmement hétérogènes qui ne laissent pas surgir un axe de conflit, leur absence d'articulation avec des identités collectives préalables, les réseaux d'emprise qu'ils tissent, les opportunités qu'ils offrent, leur compatibilité avec le maintien au moins formel de l'Etat de droit. De la banalité de la violence à la terreur, il y a à la fois continuité et rupture. Je prétends montrer que la continuité est assez forte pour que la terreur soit largement ressentie comme une situation de fait qui ne remet pas en cause les routines de la violence. Il n'y a pas de catégorie intellectuelle ou politique pour l'appréhender, encore moins pour lui donner un sens.

5 Je ne reviendrai pas ici sur le contexte dans lequel la violence actuelle s'est déclenchée ${ }^{4}$. Dans une première partie, je traiterai des interférences entre les multiples phénomènes de violence. Dans une seconde partie, j'examinerai divers aspects de la banalité de la violence. Dans une troisième partie, je considérerai les conditions dans lesquelles s'effectue le passage à la terreur. Dans une dernière partie, je considérerai les raisons pour lesquelles la terreur, par ailleurs bien réelle, ne parvient pas à dessiner une histoire ni à être mise en intrigue ${ }^{5}$, voire seulement susciter une large indignation morale.

Les interférences entre les phénomènes de violence

6 Les phénomènes de violence, je l'ai dit, sont singulièrement hétérogènes. Confrontations entre guérillas et forces de l'ordre, actions des forces paramilitaires et des narcotrafiquants, exactions des milices urbaines, opérations de " nettoyage social ", assassinats politiques, criminalité organisée et petite délinquance, disputes entre bandes de jeunes, règlements de compte et rixes individuels : autant de formes diverses qui concourent, dans des proportions diverses à l'explosion du nombre des homicides.

Dans cet ensemble, la violence proprement politique n'occupe, apparemment au moins, qu'une place limitée. En 1987, des analystes estimaient qu'elle n'était responsable que de $7 \%$ des victimes ${ }^{6}$. Mais comment fixer encore les frontières entre ce qui relève du politique et ce qui relève d'autres dimensions ? On peut estimer que les narcotrafiquants ne sont pas des acteurs politiques. Mais lorsqu'ils interviennent par la corruption dans les processus électoraux et dans les décisions judiciaires, ne deviennent-ils pas des protagonistes politiques et, a fortiori, lorsqu'ils usent massivement de la terreur pour faire plier l'Etat? A l'inverse, les guérillas sont à coup sûr des acteurs politiques. Mais le sont-elles encore lorsqu'elles consacrent une grande partie de leurs activités au racket et aux enlèvements et recourent parfois pour les mener à bien aux services de délinquants organisés ou de tueurs à solde ? Quant à la violence quotidienne, celle des meurtres crapuleux, des vengeances ou des règlements de compte, ne revêt-elle pas dans bien des cas un caractère politique dans la mesure où 
elle peut traduire aussi une rage sociale et résulte aussi de la défaillance des appareils juridico-policiers?

8 Une distinction aussi élémentaire que celle entre violence " organisée " et violence " désorganisée " n'est-elle pas discutable ? Des protagonistes comme les guérillas ou les narcotrafiquants n'ont jamais qu'une cohésion relative. Les guérillas sont multiples, et chacune d'elle a de nombreux fronts - plus de 60 dans le cas des FARC - qui sont loin d'avoir des pratiques et disciplines identiques. Les fameux " cartels " de Medellín, Cali, Bogota ou de la Côte atlantique n'étaient sans doute, même au moment de leur splendeur, que des coalitions relativement lâches de noyaux divers'. A présent qu'ils ont été partiellement démantelés, ces noyaux ont encore une plus grande autonomie. Dans le cas des paramilitaires ou des milices urbaines, les bandes sont souvent encore plus instables et les relations avec la délinquance plus considérables, même si depuis quelque temps l'on assiste à la formation de groupes d'envergure départementale et fortement centralisés. La délinquance proprement dite n'est évidemment pas seul le fait de quelques gangs épars ou d'individus, mais aussi de vastes organisations aux ramifications nombreuses. Les policiers eux-mêmes en donnent l'exemple qui, pendant un temps, ont géré avec un grand savoir-faire le trafic des voitures volées. La corruption, qui affecte toutes les organisations et les secteurs sociaux, réduit encore plus la possibilité des distinctions tranchées. Les chiffres tendent du reste à suggérer une corrélation entre la présence de protagonistes " organisés ", y compris des guérillas, et la diffusion de la violence " inorganisée ".

Une des raisons pour lesquelles les frontières entre violence politique et non-politique de même qu'entre violence organisée et violence désorganisée sont devenues de plus en plus poreuses est que tous les protagonistes détenant une capacité d'action armée visent désormais, au titre de moyen ou de but, à contrôler les pôles de production économique du pays.

L'expansion de l'économie de la drogue, celle de la marijuana dans les années soixantedix, de la cocaïne depuis 1975 et à présent celle de l'héroïne, a eu un rôle essentiel dans le déplacement des enjeux de la violence. Les deux dernières productions notamment se sont implantées dans des régions déjà tenues par une des guérillas, les FARC, ou dans lesquelles celles-ci n'ont pas tardé à s'installer. L'existence d'une guérilla de longue tradition assurait la protection des intérêts des narcotrafiquants, cultures et laboratoires étant à l'abri des incursions des forces de l'ordre. En retour, les FARC bénéficiaient soudain d'une manne financière grâce aux taxes prélevées tant sur les cultivateurs que sur les intermédiaires du trafic. C'est ainsi qu'en quelques années, au tournant des années 1980, cette guérilla pouvait doubler le nombre de ses fronts et de ses effectifs. L'essor de la culture du pavot depuis 1990 s'est effectué largement à son initiative.

Du contrôle de l'économie de la drogue, la lutte s'est ensuite étendue à celui de la plupart des pôles de production des biens primaires. Une autre organisation, l'ELN, presque anéantie au milieu des années soixante-dix, renaît ensuite de ses cendres grâce au contrôle qu'elle exerce dans les régions productrices de pétrole, et aux prélèvements financiers qu'elle peut y opérer. Il en a été progressivement de même avec les autres pôles miniers, nickel, or, charbon, comme avec ceux de l'agriculture commerciale, de la production de la banane dans l'Urabá, de la palme africaine ou les nombreuses régions d'élevage. Racket et enlèvement sont devenus des pratiques généralisées. Les zones de culture du café, longtemps restées relativement à l'abri des protagonistes de la 
violence, subissent désormais l'emprise des narcotrafiquants et des guérillas en même temps qu'elles connaissent un niveau déconcertant de violence désorganisée. Quant à la zone des émeraudes, les vastes groupes d'" autodéfense " qui y sont installés depuis toujours, les mettent à l'abri des guérillas mais pas de la violence ${ }^{8}$. De manière générale, la carte de l'implantation des guérillas coïncide avec celle des pôles de production primaire, de même que celle de la violence rurale tant organisée que désorganisée ${ }^{9}$.

Le fait que les guérillas se soient assigné pour objectif prioritaire de contrôler ces pôles économiques a eu pour conséquence de transformer les interactions entre tous les protagonistes ayant une capacité armée. Dans les zones de culture et de transformation de la drogue, une certaine coopération entre guérillas et narcotrafiquants est indispensable. Mais il faut aussi la collaboration implicite des autres acteurs présents localement, militaires, policiers, politiciens. Les transactions ne sont évidemment pas exemptes de conflits. C'est apparemment à la suite du non-respect d'un contrat tacite entre FARC et trafiquants que s'est enclenchée une confrontation sans merci entre les premières et les bandes paramilitaires mises sur pieds par Gonzalo Rodriguez Gacha ${ }^{10}$. Ce genre de litiges peut aussi intervenir entre guérillas et forces de l'ordre lorsque celles-ci exigent des commissions excessives ${ }^{11}$.

Si guérillas et narcotrafiquants doivent coopérer dans les zones de production de la cocaïne, ils ont des intérêts opposés ailleurs. A mesure que les narcotrafiquants investissent dans la propriété foncière et dans l'élevage - on estime qu'ils ont acquis plus de cinq millions d'hectares des meilleures terres -, ils se convertissent, comme les autres grands propriétaires, en une cible pour les guérillas qui entendent les soumettre aux taxes et réquisitions révolutionnaires. La confrontation prend donc dans ces régions un caractère systématique. Dans les autres pôles de production de richesses, transactions et conflits se juxtaposent également. Les guérillas n'ont nulle part paralysé la production, comme si elles étaient soucieuses de préserver leurs sources de financement. Elles peuvent protéger les compagnies et les propriétaires qui sont " en règle " avec les impôts qui leur sont fixés. La corruption du personnel politique par les narcotrafiquants et les pressions auxquelles les guérillas le soumettent constituent d'autres modalités de transaction ${ }^{12}$. Ainsi se redéfinit le cadre des interactions stratégiques sur la base d'interférences d'une grande diversité et fluidité.

Le résultat en est la fragmentation du territoire en fonction de l'emprise relative de tel ou tel protagoniste. Les interférences entre protagonistes armés conduisent à un redécoupage territorial en fonction des frontières invisibles qui s'instaurent entre les zones situées principalement dans l'orbite de l'un ou de l'autre. Des régions ont acquis une identité particulière du seul fait d'être placée sous leur emprise ou bien d'être le théâtre de leurs rivalités, comme l'Urabá et le Bas Cauca.

Il s'ensuit que la violence acquiert un caractère singulièrement prosaïque. Les idéologies politiques ou les croyances ont peu de place dans les affrontements. Certes, les guérillas ne cessent pas d'être un acteur politique. Leur puissance militaire, qui leur permet de faire acte de présence dans la moitié des municipes du pays et jusqu'aux abords d'une ville comme Bogota, suffit à leur assurer ce statut ${ }^{13}$. Elles ne bénéficient pourtant plus que d'une crédibilité politique des plus réduites. Depuis 1985, leur aura n'a cessé de pâlir, et l'opinion manifeste une exaspération croissante envers une lutte marquée par toutes sortes d'exactions et qui ne semble mener nulle part. Ce sont du reste des guérillas qui, bien avant la fin de la guerre froide, ont cessé de trouver les mots pour dire ce qu'elles voulaient et faire miroiter un avenir meilleur. Elles restent 
muettes comme si leurs pratiques actuelles suffisaient à témoigner de ce qu'elles étaient et de ce qu'elles voulaient. Or le racket n'a jamais suscité les grandes passions politiques. Là même où elles sont le mieux implantées et disposent d'un pouvoir de conviction fort, elles hésitent désormais à se mesurer directement sur le terrain électoral, à cause du climat de terreur, certes, mais aussi de la crainte de ne pas être suivies par leurs sympathisants supposés ${ }^{14}$.

La différence entre violence organisée et violence désorganisée n'est sans doute pas annulée. Mais elles entrent en résonance, et c'est ce qui conduit à la violence généralisée. Traversant aussi bien les rapports sociaux que les relations interindividuelles, influant sur le fonctionnement institutionnel autant que sur les valeurs sociales, la violence généralisée n'a plus affaire à un contexte qui lui serait extérieur et n'admet plus l'intervention d'aucun tiers. Elle suscite, à partir des interférences entre toutes les formes de violence, son propre contexte, ses propres modes de transaction et de confrontation. Elle ne s'étaye ni sur les oppositions de classe ni sur des identités collectives.

Les tensions sociales ne disparaissent pas. On en trouve dans chaque région. Elles sont même sans doute plus vives qu'elles ne l'ont jamais été. L'économie du café, qui avait puissamment contribué à la stabilité du régime colombien est plongée dans une crise sans issue. Le reste de l'économie agricole ne se porte guère mieux depuis que l'ouverture économique, réalisée sans précaution en 1991, la soumet totalement à la concurrence des produits d'importation. Le chômage urbain ne cesse lui-même de progresser depuis deux ans. Les salaires stagnent ou régressent. La fin du miracle de la drogue contraint de surcroît le gouvernement à prendre des mesures d'austérité. Tout contribue ainsi à exacerber une conflictualité latente. Ce sont les acteurs sociaux qui font défaut. Ils n'ont cessé de se décomposer davantage, pour des raisons communes à bien d'autres pays, mais aussi sous l'effet de la violence. Tantôt ils sont instrumentalisés par les protagonistes armés, tantôt ils sont emportés par la violence. Les plantations de bananes de l'Urabá offrent l'exemple d'une situation où, malgré l'intensité des tensions sociales, les acteurs sociaux ne sont jamais parvenus à s'affirmer. Pendant une longue période, les syndicats sont en butte à une telle répression qu'ils demeurent d'une extrême faiblesse. A partir de 1985, ils acquièrent au contraire une puissance de négociation apparemment considérable puisqu'ils concluent des conventions collectives qui sont parmi les meilleures de la Colombie. Mais ils sont en réalité pleinement subordonnés aux deux organisations de guérilla implantées dans la région et vont de fait vite s'affaiblir, d'autant qu'ils subiront directement le contrecoup de la guerre fratricide entre les deux guérillas en même temps que de la terreur mise en œuvre par les paramilitaires ${ }^{15}$.

Les identités culturelles résistent encore moins que les intérêts sociaux. Elles ont, il est vrai, toujours été fragiles dans un pays de métissage et de migration. Sans doute, existe$\mathrm{t}$-il des différences culturelles régionales relativement sensibles. Elles peuvent alimenter des préjugés, elles n'entrent guère en compte dans la violence. La seule exception est peut-être constituée par les régions à forte population indienne, le Cauca notamment, où s'est du reste créée une organisation de guérilla spécifique, le Quintin Lame. Encore est-il difficile de dire ce qui revient dans ce cas à une conscience identitaire et ce qui relève d'une habile utilisation du thème identitaire au service d'autres revendications. Dans le reste de la Colombie, les seules identités durables sont les identités partisanes, mises en place au XIXème siècle, remarquablement stables 
depuis, encore exacerbées par les traces de la Violencia dans les années cinquante. Ces identités ont à l'évidence de fortes assises. Mais le contenu de celles-ci dépend des rapports qui s'établissent avec les pouvoirs locaux. Autant que les identités comptent les allégeances et celles-ci se transfèrent aisément d'un chef ou d'un clan à un autre. Même les vieilles identités partisanes ne font donc pas nécessairement obstacle à l'installation des réseaux de pouvoir dépendant des protagonistes armés. La crise des partis traditionnels la rend encore plus aisée.

La banalité de la violence : carrières individuelles et logiques de protection

La notion de violence généralisée vise aussi à suggérer que, dans un premier temps au moins, la violence n'est pas vécue comme une guerre ou une catastrophe, moins encore comme le produit d'un ensemble de conduites déviantes. Elle apparait comme un processus banal, offrant des opportunités, suscitant des accommodements, comportant des normes et régulations. La banalité ne concerne donc pas seulement le profil personnel de ceux qui sont impliqués dans la violence, mais le fait que celle-ci se traduit par des interactions qui ne paraissent pas en totale rupture avec les interactions habituelles ni ne donnent naissance à de nouvelles représentations ou à un nouvel imaginaire.

La violence n'a, jusqu'à tout récemment, nullement entravé le marché de l'économie ${ }^{16}$. La Colombie peut même se targuer d'avoir surmonté la " décennie perdue " mieux que tous les autres pays d'Amérique Latine, Chili compris. L'essor de l'économie de la drogue a sans doute créé maintes distorsions, mais a par ailleurs contribué à éviter les déséquilibres de la balance des paiements. La violence altère partiellement le fonctionnement du marché en le soumettant à des rapports de force et à des contrats échappant à la légalité. Ce n'est cependant pas une situation complètement nouvelle : la précarité de l'Etat a encouragé déjà auparavant l'expansion des activités illégales, contrebande, protections politiques, clientélisme interférant avec le cours de l'économie ${ }^{17}$. Certes la violence engendre des coûts de transaction supplémentaires mais ils sont compensés par l'abondance des disponibilités en capitaux. Les propriétaires fonciers traditionnels peuvent être incités à vendre leurs terres aux narcotrafiquants, mais ils le font souvent à très bon prix, et les grands entrepreneurs industriels ou miniers peuvent être victimes du racket mais en reporter les coûts sur les clients. La poursuite de la croissance et l'impuissance de l'Etat à assurer une protection minimale poussent chacun à préférer la voie du free rider et à reculer devant les aléas d'une éventuelle action collective ${ }^{18}$.

Il est remarquable que même les milliers d'enlèvements n'ont eux-mêmes pas conduit, jusqu'à tout récemment, à une protestation massive. Tous les protagonistes armés y recourent. Toutes les victimes potentielles s'efforcent de se prémunir contre ce danger, soit en recourant à des systèmes sophistiqués de protection, soit en s'efforçant de contracter des formes complexes d'assurance. Ces stratégies individuelles semblent plus rationnelles que les protestations symboliques. De plus, la quantité des enlèvements en fait une routine qui ne surprend plus. Même si beaucoup se terminent tragiquement, ils sont considérés comme une composante supplémentaire de la violence. Chacun est condamné à s'adapter à cette éventualité. L'on peut citer le cas d'un homme politique qui, séquestré plusieurs mois par les FARC et contraint à payer une forte rançon, concluait à l'occasion de l'élection suivante une alliance avec l'Union Patriotique, le parti soutenu par les FARC. 
22 L'illégalité et la violence engendre par ailleurs de nouvelles opportunités. Cela est évident pour toutes les activités associées à l'économie de la drogue. On estime à plus d'un million le nombre de personnes qui en vivent directement ou indirectement et l'on a vu que bien d'autres souscrivaient aux perspectives de mobilité sociale qui l'accompagnent. Il ne s'ensuit évidemment pas que toute la population bénéficie des retombées de l'économie illégale et des mécanismes de violence. Certains travaux récents, partant du constat de la coïncidence entre pôles de production de richesse et violence, en déduisent que la violence est liée à la prospérité, affirmation quelque peu simpliste qui néglige les masses démunies qui y affluent et qui ne sont pas invitées au festin. D'autres, qui évoquent les abondants moyens financiers captés par les guérillas, en viennent à conclure que la violence serait une stratégie sauvage de redistribution des revenus. Tout suggère au contraire qu'à la faveur de la violence, l'inégalité, qui avait eu tendance à s'atténuer entre 1978 et 1985, va à nouveau s'accentuant. L'économie de la violence a ses exclus.

23 Il reste que la violence fournit effectivement des possibilités de carrière qui attirent notamment les secteurs les plus jeunes de la population.

24 A bien des égards, ces carrières peuvent paraître une variante parmi toutes celles offertes par le vaste champ de l'informel. Selon les estimations d'un économiste, le niveau moyen des rémunérations dans le secteur informel a progressé de 10,5\% entre 1984 et 1992 alors que celles du secteur formel ne progressaient que de 3,1\%. Les avantages assurés par le niveau d'éducation ont été en diminuant. En revanche, la rémunération moyenne des activités délictueuses a triplé entre 1980 et 1993. Il n'est donc pas surprenant qu'un pourcentage croissant de jeunes renoncent à la scolarisation pour se lancer dans les activités illégales. L'impunité est pratiquement totale dans le cas des crimes en raison de l'inefficacité de la justice : un homicide sur trois donne lieu à l'ouverture d'une enquête, quatre homicides sur cent donnent lieu à une condamnation. La réussite des grands entrepreneurs illégaux constitue une incitation supplémentaire à tenter sa chance dans la criminalité. Encore plus attirante peut être une carrière dans la subversion. Le code pénal de 1980 établit des peines réduite pour les assassinats " politiques " par rapport aux assassinats ordinaires, les peines allant de 3 à 6 ans19.

25 Entrer dans les guérillas ou dans les formations paramilitaires est une carrière comme une autre. Les unes et les autres ne donnent pas seulement une solde et des moyens de subsistance. Elles font accéder à un statut, dont l'uniforme et les armes sont le symbole. Elles permettent surtout de faire partie d'une organisation, et c'est peut-être là l'essentiel. La dislocation de la famille et la désorganisation sociale confèrent un attrait complémentaire au fait de participer à des groupes définissant des modèles de conduite et de discipline, l'autorité des commandants se substituant avantageusement, pour des jeunes qui n'ont souvent que quinze ans ou même moins, à celle, défaillante, des pères. Cela vaut tout autant pour les milices urbaines qui regroupent les jeunes du quartier. Le choix de l'entrée dans les guérillas ou les paramilitaires représente aussi souvent une alternative à la prestation du service militaire. Nombreuses sont les familles dont les enfants se répartissent entre l'armée, la guérilla ou toute autre organisation.

Les carrières sont loin d'être toutes rectilignes. Après vingt ans de violence, l'usure du temps produit bien des évolutions. Du travail au service des narcotrafiquants à l'engagement dans les paramilitaires ou au glissement dans la délinquance diffuse, la transition est facile. Des milices politiques à la criminalité, elle ne l'est pas moins. Le 
devenir des guérilleros n'est pas moins diversifié. Certains peuvent être détachés un temps auprès de narcotrafiquants. Il y a tous ceux qui reviennent à la vie civile. Il y a aussi ceux qui changent de camp en rejoignant les groupes paramilitaires. Ces cas, les plus intéressants, ne sont nullement exceptionnels. Un pourcentage important des chefs et des membres de ces groupes provient de la guérilla. Le passage peut s'opérer au moment où une zone tombe sous l'emprise des paramilitaires, mais il peut aussi se réaliser en fonction de circonstances individuelles, par exemple l'éventualité de subir une sanction ou les menaces associées aux rivalités entre guérillas. Les phases de terreur locale ne font qu'accentuer ces reconversions. En tout état de cause, la diversité de ces trajectoires atteste que le prosaïsme de la violence va de pair avec une certaine circulation des individus entre les multiples champs d'activité illégale, para-légale et légale. Ce que les élites pratiquent à leur manière par le biais de la corruption.

Par ailleurs, la présence locale d'un protagoniste armé n'obéit pas toujours à une logique de confrontation. Elle peut répondre d'abord à une logique de protection. Dans un livre sur le phénomène mafieux en Sicile, D. Gambetta présente celui-ci comme un système de protection assurant, dans des conditions de défiance, les conditions de transactions fondées sur la confiance ${ }^{19}$. Le raisonnement peut, au moins dans certains cas et certaines limites, être appliqué aux réseaux armés colombiens qui exercent leur emprise sur des territoires définis. Il ne manque pas d'endroits où les habitants, inquiets de l'expansion de la violence désorganisée, se montrent disposés à en appeler à un protecteur capable d'imposer une loi. La situation s'est souvent produite dans les zones de culture de la drogue. L'afflux de colons attirés par la perspective de gains rapides s'est traduit, au début des années 1980, par la prolifération des homicides et la présence des FARC a donc été bien acceptée. Imposant ses codes de justice et de sanction, définissant les conditions et les limites de l'accès à la terre ${ }^{20}$, veillant à ce qu'une partie des surfaces demeure consacrée aux cultures vivrières, contrôlant les transactions avec les intermédiaires des trafiquants, mettant à l'abri des incursions militaires, les FARC satisfaisaient à la fois la demande d'ordre et la défense des intérêts des cultivateurs et palliaient l'absence de l'Etat. Les guérillas n'ont pas le monopole de ce genre de protection. Dans le Moyen Magdalena, les groupes paramilitaires, qui ont remplacé les FARC vers le milieu de 1985, remplissent la même fonction au profit de ceux qui n'ont pas été condamnés à fuir. Dans les quartiers périphériques de nombreuses villes, soumis à l'arbitraire des sicarios et autres délinquants et exposés à l'anomie, les milices populaires ont pu accomplir le même rôle en assurant la surveillance locale.

L'allégeance au réseau qui contrôle le territoire est souvent la condition de l'accès au marché du travail. Le réseau instaure une manière de closed shop union. Dans l'Urabá, telle finca bananière " appartient " aux FARC, telle autre à l'EPL. Le cas se produit aussi dans les mines d'or, de nombreux domaines d'élevage, voire certaines entreprises industrielles situées dans les villes encadrées par les guérillas ou les paramilitaires. Y travailler impose de souscrire aux normes du réseau. Les sabotages à répétition menés par l'ELN contre les oléoducs sont une manière de créer des emplois : l'ELN sélectionne à l'avance la main œuvre qui sera embauchée pour nettoyer le pétrole répandu.

L'acceptation de la protection n'implique pas nécessairement une adhésion idéologique. Toute une gamme de relations s'établit. Elle va de la participation active de certains auxiliaires, tels les miliciens dotés d'armes légères ou les collaborateurs civils déclarés ou non déclarés, à la simple sympathie ou même à l'adaptation passive. 
Lorsqu'un protagoniste tient un territoire depuis des lustres, comme la guérilla dans diverses zones, des habitudes se créent et les habitants en viennent à percevoir souvent le monde extérieur comme hostile. Mais, si tous doivent se plier aux règles, beaucoup peuvent maintenir un rapport principalement instrumental avec le " protecteur ". Les stratégies individuelles d'adaptation sont variables, mais il n'est pas rare que les habitants se livrent à un calcul rationnel qui prend en compte les avantages de leur maintien dans la zone et le coût de l'obéissance qui leur est exigée. Le cas échéant, nombre d'entre eux peuvent entériner un transfert d'allégeance d'un protecteur à l'autre, comme cela s'est produit dans la zone du Moyen Magdalena citée plus haut.

Si la protection n'interdit pas les stratégies individuelles d'adaptation, elle empêche dans tous les cas les formes d'action collective autonome. J'ai déjà mentionné l'affaiblissement des acteurs sociaux. Mais le constat vaut pour toute expression de solidarité traditionnelle. Dans les zones de colonisation, les habitants étaient accoutumés à coopérer entre eux pour construire des équipements de première nécessité. Les Juntas d'Accion Comunal disposaient d'un prestige certain. Ces formes d'action commune tendent à disparaître car ceux qui en prendraient l'initiative risquent fort d'être condamnés soit à rallier les rangs du protagoniste armé, soit à s'exposer à des sanctions. Des zones où l'argent ne manque pourtant pas présentent ainsi un état d'abandon surprenant. Quant aux Juntas d'accion Comunal, elles sont de plus en plus placées sous la tutelle directe des organisations armées. Certes, les régions placées sous " protection " peuvent être le point de départ de grandes mobilisations collectives. C'est ainsi que d'amples marches paysannes se sont déroulées en 1987-88 et se sont à nouveau produites en 1995-96. Ces marches sont cependant parrainées par les guérillas, l'ELN dans le premier cas, les FARC dans le second. La participation y est rien moins que volontaire. Sans doute les paysans s'y rallient volontiers lorsqu'ils considèrent qu'elles sont destinées à promouvoir leurs intérêts. L'enthousiasme retombe quand les marches se succèdent les unes aux autres, avec les risques et les souffrances qu'elles entraînent, et que la contrainte préside de plus en plus à leur déroulement.

31 Ce système d'encadrement ne présente pas un caractère totalement inédit. Les partis traditionnels n'agissaient pas autrement dans de nombreux municipes de la Colombie. Les factions ou clans qui y exerçaient le pouvoir contraignaient souvent la population à leur faire allégeance : c'était le prix à payer, non seulement pour avoir accès aux ressources, mais parfois pour continuer à vivre en paix, voire pour éviter d'être obligé de partir. Certains auteurs parlent du reste de " clientélisme armé " pour souligner la continuité avec les clientélismes anciens. Reste la différence, elle n'est pas mince, qui résulte de l'insertion ou non dans le champ de la politique institutionnelle.

C'est dire que le quadrillage du pays par les protagonistes armés et leurs réseaux d'emprise peut être vécu comme une situation banale. Il reste que l'on ne peut en rester à la logique de la protection comme si elle répondait purement et simplement à une demande et parvenait à établir un dispositif de confiance stable. Au raisonnement de D. Gambetta, de nombreux spécialistes de la mafia sicilienne ont objecté que " l'offre " de protection importait sans doute davantage que la demande, que cette offre passait par l'usage, effectif ou potentiel, de la violence et que, loin de mettre fin à un état de défiance, elle ne cesse de l'alimenter ${ }^{21}$. A plus forte raison en va-t-il ainsi dans le cas colombien où les réseaux n'ont pas une implantation séculaire et, par ailleurs, sont en concurrence les uns avec les autres. 

interférences entre ses protagonistes. La dimension de " l'offre ", avec la violence qu'elle suppose, est au moins aussi importante que celle de la demande. Le cas des zones de culture de la drogue et de son acceptation de la règle des guérillas est loin d'être général. Nombreux sont les cas où l'imposition de la protection ne répond à aucune demande. Lorsqu'un " protecteur " remplace un autre protecteur dans une région, la population n'est guère consultée. La multiplicité des réseaux implique par ailleurs la délimitation de frontières complexes et fluides. Les frontières entre réseaux sont en effet loin de passer entre des régions facilement identifiables. Elles traversent les régions, les villes, les quartiers. Une ville importante comme Barrancabermeja, l'un des centres du raffinage pétrolier, est ainsi divisée entre quartiers paramilitaires, ELN, FARC etc. Les frontières peuvent même séparer les pâtés de maisons d'une même ville. Telle est la situation dans bien des quartiers de Medellín ou de Bogota où l'emprise de chaque milice peut ne pas aller au delà d'un micro-quartier. Dans l'Urabá, les frontières isolent les fincas les unes des autres en fonction de leur affiliation. La territorialité n'est donc pas séparable de l'action des protagonistes armés. La relation inverse peut d'ailleurs parfois prévaloir, dans laquelle l'emprise sur un territoire vient en premier, l'accumulation de pouvoir, y compris armé, en résultant. Tel est le processus par lequel sont souvent passées les milices urbaines. En établissant leur contrôle sur un quartier ou un pâté de maisons, elles préparent leur reconnaissance et peuvent entrer dans le jeu des interférences et transactions des protagonistes de la violence. La contrepartie de la logique de la protection est en tout cas la transformation du territoire en un patchwork de micro-territoires placés sous la tutelle d'une organisation.

La violence inhérente à la territorialité peut rester dans les limites de la banalité et de l'ordinaire. Il faut cependant peu pour qu'elle bascule dans la terreur.

\section{La terreur}

Comme dans le cas de la violence, il convient de distinguer entre deux types de terreur. Le premier n'a pas de soubassement territorial, le second s'y réfère explicitement. Le premier n'a rien à voir avec la logique de la protection. Le second en est l'expression pervertie. C'est le second type que j'analyserai davantage. Il convient cependant de considérer d'abord brièvement le premier.

Les narcotrafiquants mettent souvent en œuvre une terreur territorialisée, par l'intermédiaire des paramilitaires. La terreur à grande échelle qu'ils ont pratiquée de 1987 à 1993, celle qui a eu le plus de retentissement, n'a en revanche aucun rapport avec une problématique territoriale. Elle vise l'Etat et l'opinion afin que la déstabilisation de l'un et le désarroi de l'autre amènent à renoncer aux mesures d'extradition. Les attentats ciblés contre des personnalités de premier plan, hommes politiques ou magistrats, de même que les attentats aveugles à la voiture piégée ou par d'autres moyens ${ }^{22}$, n'ont, au premier abord du moins, pas d'autres motifs. Le recours à de telles méthodes est sans doute facilité par la banalisation de la violence. Dès 1984, le cartel de Medellín a pu tuer un ministre de la Justice sans qu'il en résulte un émoi durable et sans que cela empêche, aussitôt après, des notables d'entreprendre des négociations discrètes avec lui. Mais ce recours traduit aussi une inflexion inédite. Pour la première fois, un protagoniste de la violence entreprend de déstabiliser l'Etat. Dans la longue histoire de la violence antérieure, rien de tel ne s'était jamais produit. Des personnages de premier plan ont pu être assassinés, comme Gaitan en 1948, et d'autres contraints à l'exil ${ }^{23}$, mais les protagonistes ne s'attaquaient pas au fonctionnement de 
l'Etat lui-même. Les guérillas se sont du reste longtemps targuées de rejeter tout terrorisme. Une sorte d'interdit a été levé et le champ de la violence connaît de ce fait une redéfinition.

La terreur déployée contre les militants de l'Union Patriotique et autres activistes syndicaux ou politiques ne s'insère pas non plus dans une problématique proprement territoriale. Elle a une finalité principalement politique. L'alliance entre narcotrafiquants, militaires et dirigeants politiques locaux vise avant tout à éliminer une opposition politique constituée dans la mouvance de la guérilla. Elle a, il est vrai, des finalités annexes de la part des narcotrafiquants, comme celle de desserrer la pression exercée par les guérillas sur leurs possessions. Mais la pratique systématique d'une terreur politique déterritorialisée représente bien une autre innovation par rapport aux modalités anciennes de la violence.

Les militaires se voient imputés par les populations paysannes une part majeure dans la production de la terreur. Les excès innombrables qu'ils commettent au cours de leurs opérations ne suffisent pas à expliquer cette mise en accusation générale. Le fait que, pour beaucoup, ce soit la seule occasion où ils aient un contact avec " l'Etat " et que cet Etat n'a pas précisément la figure qu'ils supposaient compte sans aucun doute. Mais cela tient surtout à ce que les militaires procèdent par incursions ponctuelles, n'occupent pas le terrain et se soucient fort peu de constituer des réseaux de protection. Le fonctionnement passablement bureaucratique de l'armée n'offre pas la possibilité à ses cadres, constamment déplacés, de se familiariser avec les habitants. Non seulement ils frappent souvent à l'aveugle, assimilant quand cela leur convient paysans et guérilleros, mais ils laissent à nouveau, lorsqu'ils se retirent, le champ libre aux guérillas. Les individus ne disposent donc d'aucune marge pour des stratégies d'adaptation. Les militaires couvrent par ailleurs les paramilitaires auxquels ils délèguent en fait la fonction tant de réaliser la plupart des massacres d'envergure que de s'implanter territorialement. Les policiers urbains ont une réputation bien pire encore. Durant la " guerre " contre le cartel de Medellín, ils ne procédaient pas autrement que les militaires dans les régions rurales, faisant de brutales irruptions dans les quartiers soupçonnés d'abriter les sicarios et tuant ou torturant souvent au hasard. Il n'est guère douteux que ces abus des forces de l'ordre incitent la population à entériner les contraintes et excès des autres protagonistes, au moins tant qu'ils ne dépassent pas la limite de l'insupportable.

J'en viens ainsi à l'autre type de terreur, celle liée aux interactions entre réseaux et à l'emprise territoriale. J'ai mentionné précédemment la complémentarité de la protection et de la violence. Même en dehors des disputes entre protagonistes, la violence banale peut aisément devenir terreur.

La prolongation de la violence entraîne dans bien des cas une dégénérescence des protagonistes armés qui ne se traduit pas seulement par les exactions ou la corruption. Il en va ainsi pour bien des organisations de narcotrafiquants et, dans sa dernière étape, le cartel de Medellín s'est livré à de nombreux règlements de compte internes. Les paramilitaires et les guérillas n'ont pas été davantage épargnés par les épisodes de justice sommaire. Chaque guérilla a connu ses processus d'épuration sanglants. Dès les années soixante-dix, le leader de l'ELN, Fabio Vasquez Castaño avait donné l'exemple en fusillant la plupart des cadres d'origine universitaire qui avaient rallié l'organisation. Les FARC ont réussi à maintenir un plus grand secret sur ces liquidations. Elles ont cependant été nombreuses et constantes, menées soit 
directement par le Secrétariat central, soit confiées aux commandants des blocs et fronts, et l'on sait par exemple que Braulio Herrera, chargé à la fin des années quatrevingt de récupérer la région du Magdalena Medio, a procédé à des exécutions massives qui lui ont valu d'être envoyé à l'extérieur. Tout récemment encore, à l'occasion de la guerre dans l'Urabá avec les paramilitaires, le commandant de l'un des fronts des FARC donnait l'ordre d'exécuter tous les hommes qui ne feraient pas preuve d'une combativité suffisante. Le cas le plus sinistre est celui du front Ricardo Franco, dissidence des FARC et longtemps proche du M19, dont les deux leaders ont exécuté en 1987 à Tacueyo la quasi-totalité de leurs troupes, plus de 200 personnes, sous prétexte qu'elles seraient infiltrées par des agents secrets. Le choc provoqué par ce massacre n'a du reste pas été étranger à la décision $d u \mathrm{M}^{24} \mathrm{~d}$ 'entamer une négociation avec le gouvernement et à la perte de crédibilité des guérillas.

Si la terreur peut sévir à l'intérieur des organisations armées, elle touche plus encore la population. Les exactions indiscriminées pratiquées au début des années quatre-vingt par le front des FARC implanté aux alentours de Puerto Boyaca, les plus pauvres étant également soumis à des impôts exorbitants et soumis aux enlèvements à des fins de rançon, ont conduit une grande partie de la population à se rallier aux paramilitaires et à se plier à leur protection, par ailleurs étayée sur la peur et la délation. Les réseaux de protection impliquent l'existence d'informateurs prompts à dénoncer tous les " suspects ". La loi du silence s'impose vite au sein de populations qui apprennent à ne faire confiance à personne. Le franchissement des frontières du réseau, serait-ce pour les activités commerciales les plus ordinaires, peut devenir motif à accusation.

La terreur peut être encore accrue par une dimension d'incertitude. Je viens de rappeler le cas du changement d'obédience de la zone de Puerto Boyaca. Mais il y a aussi celui des déserteurs qui changent de camp. C'est désormais une expérience assez fréquente pour que la population apprenne à se défier de la protection des réseaux apparemment les plus solides car les déserteurs emportent des informations qui permettent, au cas où la zone serait obligée de changer d'affiliation, des sanctions sans merci. On dispose à ce propos d'indications précises sur une petite bourgade du Santander, La India, corregimiento de Cimitarra ${ }^{25}$. Un front des FARC y a longtemps régné en maître, imposant une protection non dénuée d'excès. Des désertions ont commencé de s'y produire au profit des paramilitaires, qui ont conduit le commandant à multiplier les châtiments contre la population. Jusqu'au jour où il est lui même passé du côté des paramilitaires. Toutes les conditions de la défiance sont ainsi réunies, envers le réseau mais aussi envers les voisins.

La situation de terreur est évidemment encore plus sensible lorsque le territoire devient l'enjeu du conflit entre divers protagonistes. La " protection " se convertit en moyen de guerre, les " frontières " en lieux de confrontation indiscriminée. Ce n'est pas un hasard si l'Urabá est la région où la terreur est la plus chronique et intense. Tous les protagonistes y sont présents car la région, par delà la production de bananes, occupe une position stratégique à la limite de Panama et c'est par le port de Turbo ou d'autres routes locales que transite une grande partie de la drogue et des armes. Il a pu arriver que les transactions prévalent. Longtemps, les FARC, les milices, les narcotrafiquants, les paramilitaires et leur chef, Fidel Castaño ${ }^{26}$, ont ainsi maintenu une sorte de modus vivendi à l'intérieur du port de Turbo. Mais tous les protagonistes n'ont cessé parallèlement de se livrer des guerres dont les axes ont changé à plusieurs reprises. Au début des années quatre-vingt, les propriétaires des bananeraies mènent une lutte 
implacable contre les organisations de travailleurs. De 1985 à 1987, ce sont les deux organisations de guérilla installées dans l'Urabá qui s'affrontent pour étendre leur implantation, entraînant les deux organisations syndicales dans leur conflit. A partir de 1987, les forces paramilitaires se déchaînent sous l'impulsion des narcotrafiquants et des militaires. La succession de massacres en 1988, qui visent surtout l'EPL, donnent la mesure des moyens employés. En 1991, l'EPL dépose les armes. Dès lors, les FARC et un noyau dissident de l'EPL tentent de s'emparer de tous les territoires de l'EPL. Les massacres s'enchaînent les uns aux autres, parfois au rythme d'un par semaine ou davantage, comme au mois d'août 1995, et nombre d'anciens de l'EPL sont amenés à reprendre les armes, en s'alliant souvent avec les militaires et paramilitaires. En 1995, commence pourtant la grande offensive des paramilitaires qui, sous le nom d'Association d'Autodéfense de l'Urabá et du Cordoba et avec Fidel Castaño pour leader, entreprennent la reconquête de l'ensemble de la région, délogent les FARC, obligées de se réfugier dans la montagne, et expulsent des milliers d'habitants.

L'entrecroisement et le déplacement des axes de confrontation se traduisent par la succession des épisodes d'atrocité. Il est certain que les paramilitaires sont responsables du plus grand nombre. Mais tous les protagonistes usent de la terreur et aucun n'a le monopole des massacres collectifs qui s'enchaînent les uns après les autres, parfois sous prétexte de vengeance. Tous sont susceptibles de recourir au service de sicarios pour commettre des assassinats en brouillant les pistes. Les retournements de situation favorisent les désertions, ce qui accentue encore l'insécurité. Les paramilitaires au cours de leur offensive de 1996 ont tué nombre de miliciens liés aux guérillas. Mais ils en ont incité d'autres à rejoindre leurs rangs, par la contrainte ou par l'attrait de soldes deux fois supérieures à celles versées par les guérillas. Des dizaines de guérilleros ont abandonné leur organisation. Il n'est donc pas difficile aux uns et aux autres de frapper avec précision. C'est ainsi que les bandes de tueurs se présentent souvent dans les quartiers ou les lieux de travail avec la liste des " condamnés " toute préparée. Ce qui ne les empêche pas de frapper aussi à l'aveugle. Nous avons vu que les réseaux étaient faits de couches concentriques d'affiliés. Entre le militant et celui qui n'appartient au réseau qu'en raison de son lieu de résidence, les tueurs ne font pas nécessairement la différence. La terreur est destinée à intimider toute la population.

L'intensité de la terreur dans l'Urabá ne se résume pas aux massacres et autres horreurs. Elle renvoie au fait que le patchwork résultant des rivalités entre protagonistes est fait des pièces particulièrement entremêlées. Non seulement les fincas voisines, mais les quartiers d'une même bourgade, voire les membres d'une même famille peuvent dépendre de réseaux différents. Dès lors la méfiance règne, $\mathrm{y}$ compris entre proches. La " loi du silence " n'est plus une imposition des réseaux, elle est adoptée comme règle de prudence par les individus dans leurs interactions quotidiennes. Les possibilités de stratégies individuelles d'adaptation deviennent à peu près nulles, en dehors de l'effort pour " ne rien voir " et " ne rien savoir ". Le déplacement forcé des habitants de hameaux et quartiers entiers prouve que la définition de l'adversaire est très extensive. Dans d'autres endroits, la population se voit offrir la possibilité de passer sous l'emprise du nouveau protecteur. Les paramilitaires sont du reste loin d'être dénués d'appuis locaux. Les propriétaires fonciers et la petite bourgeoisie urbaine ne sont pas les seuls à se réjouir discrètement 
de l'expulsion des guérillas et de leurs affiliés. Une partie non négligeable des secteurs populaires, exaspérée par leurs exactions et les affrontements sans fin, fait de même.

Le cas de l'Urabá est exceptionnel en ce que la guerre entre les guérillas ajoute une dimension à la violence et qu'aucun protagoniste ne peut renoncer à préserver une présence dans cette zone stratégique. Il n'est pas exceptionnel en revanche par le mélange de terreur et de protection. Le Moyen Magdalena connaît le même mélange, ainsi que bien d'autres régions. Dans les villes, les milices se changent fréquemment en bandes se livrant aux chantages et aux crimes. Elles prétendent souvent continuer à épargner leur quartier et ne frapper que les quartiers voisins. Le résultat n'est pas très différent.

Le recours à la terreur s'accompagne de mises en scènes de l'horreur pour empêcher que la population ne puisse avoir des velléités de résistance. Les armes sophistiquées ont, pendant un temps, réduit le recours aux rituels de mise à mort en usage pendant la Violencia des années 1950 où les corps étaient l'objet de mutilations codifiées avec soin $^{27}$. Les massacres collectifs relèvent d'une symbolique fondée sur le nombre et largement influencée par les séries télévisuelles nord-américaines ou mexicaines. Les sicarios de Medellín s'entraînaient du reste en imitant les gestes des héros de ces séries. Certaines autres pratiques restent cependant empruntées à l'épisode antérieur, tels l'envoi de menaces graduées ou les signes témoignant d'une mort annoncée. Mais la terreur récente, en particulier celle mise en œuvre par les paramilitaires, est marquée par le retour des anciennes pratiques. Les corps démantelés sont souvent disposés dans les lieux publics en guise d'avertissement.

Il n'y a pas de recours pour les populations soumises à la terreur. Les institutions publiques n'offrent, elles, aucune protection. Les forces de l'ordre sont souvent aux avant-gardes de la terreur et la justice, on l'a vu, brille par ses carences. L'action collective est dans ce contexte encore plus frappée d'interdit que face aux réseaux ordinaires. Des maires de l'Union Patriotique, jouissant en principe de la confiance des FARC, se sont efforcés de mener une gestion au service de la population. Menacés par les paramilitaires, ils se sont souvent aussi trouvés exposés aux exigences et aux incursions des FARC. Presque tous ont été tués. Les FARC ont souvent donné l'impression de redouter les dérives pacifistes de ces élus et de se résigner facilement à ce qu'ils se convertissent en " martyrs ". L'on peut bien citer quelques cas où des leaders locaux ont tenté d'organiser la population contre la violence et de faire reconnaître leur neutralité par les protagonistes armés. Ces tentatives ont jusqu'à présent toujours tourné court. En 1987, des dirigeants de la India, la bourgade citée plus haut, ont voulu relever le défi avec le soutien de secteurs de l'Eglise en demandant aux paramilitaires et à la guérilla qu'ils respectent leur territoire. Ils sont assassinés par les paramilitaires en 1990 et, tandis que nombre d'habitants doivent se réfugier ailleurs, la peur devient à nouveau la règle. En 1995, une femme, Gloria Cuartas, est élue maire d'Apartado, la plus grande ville de l'Urabá, sur la base d'un accord entre toutes les forces politiques, communistes compris, pour faire barrage à la terreur. On a vu ce qui a suivi : la terreur s'est encore accrue, et les paramilitaires ont montré, par un acte barbare ${ }^{28}$, le peu de cas qu'ils faisaient du maire cependant que les communistes dénonçaient l'accord. Un municipe du Cesar, Aguachica, s'est lui aussi lancé dans l'aventure de la neutralité en essayant de s'appuyer sur l'opinion internationale. Mais l'offensive des paramilitaires s'étend à présent à ce département, et assassinats et massacres se succèdent à Aguachica. 

de la violence. Ce n'est pas un hasard si l'on est amené à mentionner davantage le rôle des paramilitaires que celui des militaires. Les militaires ont apporté à plusieurs reprises la preuve de leur inefficacité opérationnelle. L'accroissement très considérable de leur budget au cours des dernières années - il a été multiplié par cinq - ne s'est pas traduit par une amélioration corrélative de cette efficacité. Les sanctions, pourtant exceptionnelles, qui ont frappé certains officiers compromis dans les atrocités ou le soutien direct aux paramilitaires, ont engendré ce que des hauts gradés eux-mêmes appellent " le syndrome du Procureur " et les incitent souvent à un attentisme prudent. Ce qui ne les empêche pas, bien au contraire, de laisser faire en sous-main les paramilitaires dont l'efficacité est autrement plus avérée. Les militaires ne sont pas les seuls à déléguer ainsi à des acteurs privés le soin de la lutte contre les guérillas. Un large consensus implicite, qui inclut notamment les associations d'éleveurs et d'agriculteurs mais aussi bien des secteurs politiques, se dessine à ce propos depuis 1995. La crise d'autorité gouvernementale et le discrédit des institutions qui en découle, ne fait que contribuer à cette situation.

Une terreur sans histoire

50 Avec la diffusion de la terreur, la banalité de la violence devrait avoir un terme. Les populations affectées se trouvent en effet confrontées avec l'expérience de l'intolérable. Les actes de cruauté et de barbarie s'insèrent toujours, peu ou prou, dans la rationalité instrumentale et stratégique des protagonistes. Mais ils témoignent aussi d'un excès qui déborde cette rationalité et qui est d'autant plus énigmatique qu'il ne renvoie pas à un antagonisme articulé à des " idéalités " ${ }^{29}$ car il reste pris dans le prosaïsme de la violence, et qu'il s'attaque pourtant aux fondements du lien social ou de l'appartenance à une commune humanité. Or il n'est pas évident que la terreur brise complètement la banalité de la violence. Il s'agit dans cette dernière partie d'en expliquer les raisons.

51 La première tient au contexte institutionnel. Celui-ci présente deux aspects contradictoires mais qui contribuent pareillement à atténuer la visibilité de la terreur.

52 D'une part, les régulations institutionnelles subissent le contrecoup de la violence. L'on a fait référence à l'inefficacité de la justice, et c'est un élément fondamental dans la banalisation de la terreur. Ce n'est cependant pas le seul. Les normes juridiques ont elles-mêmes perdu leur fonction instituante en devenant objet de transaction. Le système de remise des peines mis en place en 1991 est vite apparu comme conduisant à une négociation discrète avec les trafiquants et les peines souvent dérisoires qui leur ont été infligées, au moins pendant un temps ${ }^{30}$, ont contribué au sentiment d'impunité. En 1993, la révision du code de procédure pénale s'est faite en accord avec les avocats de ces trafiquants. De manière plus générale, la corruption du personnel politique jusqu'au plus haut niveau montre que les règles de l'informalité et de l'illégalité s'imposent jusque dans les institutions. Dès lors celles-ci paraissent entraînées dans le champ de la violence.

D'autre part, la Colombie ne cesse pas de se réclamer de l'Etat de droit. La Constitution de 1991 va même très loin dans la mise sur pieds de mécanisme de protection et d'élargissement de la vie démocratique. Des organismes de " défense des droits de l'Homme " sont installés dans toutes les institutions, y compris l'institution militaire. Si les militaires disposent d'une large liberté de manœuvre dans le choix des tactiques et moyens d'action, ils ne peuvent s'affranchir de leur subordination envers le pouvoir

Cultures \& Conflits, 24-25 | hiver 1996- printemps 1997 
civil ${ }^{31}$. Il est arrivé, on l'a vu, que des sanctions soient prises contre des gradés, y compris de haut niveau, et une épuration très vaste a été menée dans la police. Les actions menées par la Fiscalia depuis deux ans ont eu à tous le moins pour effet de mettre fin à l'acceptation sociale dont bénéficiaient les narcotrafiquants et de mettre en lumière la corruption politique. La Cour Constitutionnelle a son mot à dire sur le bien-fondé des Etats d'exception. On ne sait pas que ce genre de mesures soit habituel dans les pays latino-américains confrontés à une guerre " contre la subversion ". Le terme même de " guerre " est du reste frappé d'interdit, tant dans le langage des gouvernements que dans celui des médias et de l'opinion. Depuis 1982, les premiers ont ouvert à maintes reprises des pourparlers avec les guérillas, qui ont eu à tout le moins pour résultats, outre le renoncement aux armes de la part du M19, de l'EPL, du Quintin Lame et d'organisations moins importantes, la perte de prestige politique des guérillas toujours en activité. Quant à l'opinion, elle rejette constamment l'idée d'une guerre frontale, parfois dans l'espoir de parvenir à une solution d'ensemble négociée, plus souvent encore par scepticisme envers les possibilités d'un dénouement militaire et crainte de ses conséquences sur les espaces de liberté. Mais cette adhésion formelle à l'Etat de droit n'interrompt pas la violence. Elle lui ouvre souvent au contraire un plus grand champ, " ordre " et " violence " apparaissant comme indissociables ${ }^{32}$. Elle conduit surtout à atténuer la visibilité de la violence comme de la terreur qui prennent l'aspect d'un résidu incompressible de non-droit.

La seconde raison est que la terreur elle-même ne s'inscrit pas dans un conflit amiennemi. Une telle opposition est sans doute perceptible dans certaines zones et à certains moments. Les confrontations entre paramilitaires et guérillas revêtent incontestablement l'aspect d'une guerre sans merci et interrompent les transactions habituelles. De même traduisent-elles une incontestable polarisation sociale plus large. Mais les transactions entre les protagonistes en guerre continuent dans d'autres régions. Elles sont toujours impliquées par le fonctionnement de l'économie de la drogue. Il est possible que la rentabilité de cette économie soit bien moindre qu'auparavant, les prix internes subissant le contrecoup d'une certaine désorganisation des réseaux à la suite de l'arrestation de nombreux leaders des cartels et du détournement du trafic au profit d'autres pays, en particulier le Mexique. Les données disponibles tendent cependant à montrer que les surfaces consacrées à la culture de la coca ne diminuent pas et que celles consacrées à la culture du pavot sont en augmentation. Les FARC continuent à y être pleinement impliquées. C'est même sous leur égide et au milieu d'un petit paysannat traditionnellement sensible à l'influence de la guérilla que se développe la production d'héroïne. Le jeu à multiples facettes continue donc, trafiquants et FARC étant associés ici, adversaires là. La terreur ne remet pas non plus en cause le prosaïsme de la violence. Bien des intérêts s'abritent derrière l'intervention des paramilitaires. La terreur s'autofinance sans mal dans la mesure où, dans les régions reconquises, le prix des terres et des commerces connaît une réévaluation très notable.

Si le clivage ami-ennemi existe localement, il ne concerne que les protagonistes et leurs affiliés. Dans les zones de terreur, la population est, certes, prise dans les rapports de force. Dans son immense majorité, elle ne les déchiffre cependant pas en termes politiques. Les repères politiques ont perdu pour elle presque toute signification. Les taux d'abstention électorale en témoignent qui, à l'occasion de nombreuses élections, atteignent désormais les $80 \%$. En se contentant d'encadrer la population sans prétendre gagner son adhésion et en renonçant même fréquemment à présenter des candidats 
plus ou moins sympathisants pour soutenir ceux des partis traditionnels, quitte à les mettre ensuite sous tutelle, les guérillas prennent acte de la dévalorisation de la politique. A bien des égards, l'on a affaire à une société dans laquelle le politique moderne dans sa fonction instituante disparaît. On y retrouve à certains égards les traces de la politique du XIXème siècle fondée sur des appartenances collectives et des interactions de clientèles ${ }^{33}$. Avec la différence que les appartenances collectives et les clientèles s'étayent en beaucoup d'endroits sur la simple contrainte. Les rapports de force qui quadrillent la société sont des rapports de fait, de même que les affrontements locaux. Ils ne mettent pas en jeu un imaginaire quelconque ni ne diffusent une représentation générale d'un antagonisme politique irrémédiable.

La troisième raison réside dans la mémoire des expériences antérieures de la violence, et en particulier de l'épisode de La Violencia de 1946 à 1964. Cette mémoire présente, elle aussi, des traits contradictoires. Elle est celle d'une guerre civile entre les deux partis traditionnels et s'inscrit donc dans une vision " ami-ennemi ". Eu égard aux horreurs qui ont accompagné cette confrontation, les traces qu'elle a laissées contribuent au refus actuel du retour à une confrontation d'ensemble. Mais c'est en même temps une mémoire qui n'a pas de forme socialement reconnue. L'accord politique qui y a officiellement mis fin en 1958, avec l'instauration du Front national, impliquait qu'une chape de plomb recouvre les événements antérieurs. Tout au plus était-il admis que pendant un temps une composante de barbarie avait affleuré. Or la notion de barbarie revenait à acquitter les élites du rôle, pourtant décisif, qu'elles avaient eu dans la violence, et à imputer cette dernière à la seule immaturité des classes populaires. Il ne restait à celles-ci que le souvenir d'une humiliation. Elles s'étaient battues pour le compte des Autres, dans le cadre des allégeances qui les liaient à eux, avaient fourni la quasi-totalité des victimes, et se retrouvaient à la fin au banc des accusés. L'expérience qu'elles avaient vécue ne s'inscrivaient pas dans une histoire faisant sens. Elle restait à l'état d'une infra-histoire dépourvue d'expression.

Ce n'est pas un hasard si la mémoire de La Violencia s'exprime de trois manières à peine articulées entre elles. Elle se formule d'abord à travers la référence à l'opposition des deux partis. Mais c'est une manière de négliger les autres dimensions de La Violencia, entre autres celle des intérêts socio-économiques qui y interviennent, et d'entériner la position de subordination des classes populaires envers les élites. De plus, ces repères partisans ont à présent perdu de leur signification en sorte que les souffrances subies semblent relever de l'archaïsme. La mémoire s'exprime ensuite par des récits individuels qui ne parviennent pas à s'insérer dans un récit collectif. Ce qui traduit le caractère éclaté et local des expériences. Mais ce qui montre aussi qu'il n'y a que des points de vue séparés et immergés dans la factualité des incidents, d'où n'émerge pas une intrigue globale qui impliquerait l'inscription des points de vue particuliers dans un ou plusieurs points de vue d'ensemble. L'expérience collective ne prend forme qu'à travers une lecture mythique, et c'est là la troisième modalité de la mémoire. Les victimes affirment ainsi que la violence est présente " depuis toujours ", que celle de 1946 est la continuation de celle de 1932-33, celle de 1932-33 la continuation de la guerre des Mille Jours, la guerre des Mille Jours la continuation de toutes les guerres civiles du XIXème siècle. Ils évoquent la " Violence " comme une sorte de phénomène anonyme ou comme une catastrophe comparable aux catastrophes naturelles. Ils attribuent tout ce qui leur arrivé depuis, migration, changements 
d'activité, adoption d'autres valeurs, aux effets de la violence. La violence prend alors effectivement l'allure d'un mythe.

Cette mémoire clivée contribue à forger la manière dont les phénomènes actuels sont perçus. L'expérience de l'humiliation reste perceptible. Elle l'est dans la rage de nombre des jeunes des classes populaires qui participent, par un biais ou un autre, à la violence actuelle, en donnant le sentiment de vouloir reprendre le fil des événements antérieurs, soit pour leur donner un autre aboutissement, soit pour pousser encre plus loin dans l'infra-histoire. Leur défiance envers l'Etat et les dirigeants politiques se nourrit d'un ressentiment ancien. La division partisane, si dépassée soit-elle, laisse des traces dans la sensibilité de nombre de secteurs de la population. L'éclatement des points de vue demeure aussi perceptible. Si la violence interdit l'action collective et oblige au repli sur soi, cette individualisation négative renvoie aussi à l'héritage des épreuves de dissolution du lien social. Les représentations mythiques n'ont rien perdu de leur pouvoir. Plus que jamais prévaut la conviction que la violence du présent est la même que celle du passé et que les rapports de force sont au cœur du fonctionnement de la société.

Une telle mémoire concourt donc à ce que violence et terreur soient banalisées comme si elles étaient dans l'ordre des choses. Elle rend difficile la perception de ce qui est nouveau dans la situation actuelle et donc la mise en sens de ce qui se passe. Cette confusion n'est pas réservée aux régions récemment colonisées qui sont celles sur lesquelles l'autorité de l'Etat ne s'est jamais exercée que de façon précaire. Elle se manifeste aussi dans les régions depuis longtemps intégrées à l'économie commerciale et dans les villes.

60 L'on évoquera à ce propos l'exemple d'une importante bourgade du Nord du Valle, Trujillo, située près d'un des principaux axes routiers et l'un des pôles de la production de cafés ${ }^{34}$. Aucun cas ne peut mieux illustrer les continuités et les discontinuités des phénomènes de violence.

61 Fondé en 1931, ce municipe a d'abord été aux mains de caciques libéraux qui ont fait en sorte que la majorité des nouveaux colons appartiennent à ce parti, en les recrutant jusque dans les prisons. L'appropriation des terres, réputées publiques, mais réclamées par un notable arguant de titres de propriété de l'époque coloniale, s'est accompagnée de premiers conflits sociaux. A partir de 1942, des leaders conservateurs ont commencé à vouloir se constituer une clientèle. La Violencia leur a fourni l'occasion de parvenir à leurs fins. Massacres et terreur permettent de " conservatiser " entièrement la bourgade. La plupart des anciens habitants sont obligés de fuir ou de passer au parti conservateur. Rachetant à vil prix les terres " abandonnées ", un des leaders conservateurs acquiert une fortune et un pouvoir qui, pendant plus de trente ans, en fait le gamonal incontesté jouant un rôle jusque dans la politique départementale et nationale. Bien que la bourgade soit à $90 \%$ conservatrice, la violence ne cesse pas pour autant d'être à l'ordre du jour. Elle est désormais associée à la concurrence entre factions conservatrices. C'est par un recours incessant à la terreur que le gamonal maintient sa domination, tuant parfois lui-même des opposants, utilisant ouvertement des hommes de main pour les éliminer, contraignant leurs fidèles à fuir, maintenant un contrôle étroit sur la population. Ce qui ne l'empêche pas, encore en 1978, de recevoir l'hommage des dirigeants nationaux du parti. A partir de 1980, tous les protagonistes de la nouvelle violence font cependant acte de présence dans le municipe. L'ELN y implante un front qui gagne la sympathie de nombreux paysans. Un puissant 
narcotrafiquant, installé dans le municipe voisin, entreprend d'accaparer les terres. Les successeurs du gamonal usent de tous les moyens pour assurer leur emprise. Les militaires sont sur place. Un curé s'efforce de préserver un modus vivendi. La peur est donc en permanence présente et chacun sait que la terreur peut s'abattre d'un moment à l'autre. Trois incidents vont effectivement précipiter le drame. En 1990, arrive en plus un noyau dissident du M19 qui prétend rançonner le narcotrafiquant. A la même époque, l'ELN organise une marche paysanne vers la place de Trujillo à laquelle les paysans participent contraints et forcés. Au début de 1991, un militaire est tué dans une embuscade. Dans les jours qui suivent, les militaires, et les paramilitaires liés aux narcotrafiquants liquident les uns après les autres tous les " suspects ". Il y aura plus de 120 morts, parmi lesquels le curé. Longtemps occultés, les faits finiront par être révélés. L'Etat sera même obligé, pour la première fois, de reconnaître officiellement sa responsabilité.

Ce cas permet un certain nombre de remarques. Il est clair que les enjeux et les acteurs de la violence sont fort différents en 1930, en 1950, en 1960-70 et en 1990. Les épisodes se succèdent néanmoins avec suffisamment de fréquence pour produire le sentiment d'une continuité. Les processus de constitution sociale de la mémoire et d'inscription des faits dans une histoire sont compliqués par l'absence de points d'arrêt ou d'ancrage. La violence ouverte apparaît bien comme une composante de toute relation de pouvoir. Cette relation traverse pleinement les institutions. En 1978, après vingt ans de Front National, les assassinats perpétrés à l'initiative d'un chef local ne sont pas considérés comme des atteintes à l'Etat de droit. Les droits civils minimaux sont toujours précaires. C'est le cas du droit de propriété. L'obtention d'un titre de propriété passe par des procédures complexes et la violence enlève leur portée aux titres les mieux établis comme le sont en principe ceux des paysans des régions de café. L'univers paysan est ainsi en situation d'insécurité permanente. La citoyenneté politique n'est pas moins précaire. Rien ne la protège. De même l'identité collective est totalement hétéronome, soumise comme elle l'est à l'emprise des réseaux. Le rapport avec le gamonal n'est pas fondamentalement différent de celui avec les guérillas ou d'autres forces. Le passage de la violence ordinaire à la terreur s'effectue sans rupture majeure même si la terreur est ce qui fait événement. Il se trouve que le massacre de 1991 a été officiellement reconnu. C'est une exception. Habituellement, l'événement ne peut s'inscrire dans aucune trame solidement constituée. Dans un municipe comme celui-ci, il est difficile de parler encore de frontières même invisibles à propos de la violence actuelle. La population est en permanence prise dans les interactions entre les multiples protagonistes. La terreur locale s'insère sans doute dans l'ensemble des phénomènes de terreur au plan national. Pour ceux qui y sont immergés, cet horizon plus vaste n'a guère de signification. C'est une autre raison pour laquelle la terreur ne s'insère pas aisément dans une histoire plus vaste. Elle demeure largement irreprésentable.

Conclusion

63 Banalité de la violence, impossibilité de construire une représentation signifiante de le terreur: tels sont les deux thèmes que j'ai cherché à mettre en lumière. Ils ont plusieurs autres corrélats. J'en mentionnerai seulement deux ; l'éclatement de la notion d'individu et la dislocation de l'opinion.

64 J'ai parlé d'expérience individuelle de la violence et de la terreur. Mais de quel individu s'agit-il ? L'individu se trouve en fait à la rencontre de tensions diverses. Il est enfermé 
dans les réseaux d'emprise, voué à des stratégies de survie, sceptique envers les institutions, demandeur pourtant de " l'aide de l'Etat ". Il n'y a pas de synthèse entre ces tensions multiples. De ce fait l'identité même de l'individu, sa constance dans le temps, ne vont pas de $\operatorname{soi}^{35}$. Ce n'est pas un hasard si la seule expression de cette constance réside dans le récit d'une trajectoire qui l'amène d'une situation à une autre.

L'opinion publique n'est pas logée à meilleure enseigne. Elle réagit à l'événement lorsque celui-ci revêt une dimension symbolique majeure. Encore ces événements sontils vite oubliés, l'un chassant l'autre. De surcroît l'émotion s'émousse. En dehors de ces temps forts, l'opinion ne se manifeste guère. Sur le trafic de drogue, la politique envers les guérillas, les phénomènes de violence, la corruption, elle ne prend pas vraiment forme ou, ce qui revient au même, évolue selon les circonstances, allant d'une inclination à l'inclination contraire, de la demande de transactions tous azimuts à la demande de solutions de force. A fortiori en va-t-il ainsi à l'égard de la terreur. Car ceux qui font l'opinion n'y sont qu'exceptionnellement exposés. Les massacres en série de l'Urabá sont lointains. Les premiers qui se sont produits ont pu surprendre ; à force de se multiplier ils se transforment en simples faits divers. A mesure que la violence pénètre plus profondément jusque dans les villes, le désarroi augmente et les repères traditionnels se fragilisent. L'absence de réaction face aux avancées actuelles des paramilitaires, et à leur cortège d'horreurs, en témoigne.

La violence, on l'a vu, devient un mode de fonctionnement qui corrode les institutions elles-mêmes. L'Etat de droit, certes, continue mais sans avoir de prise, ou presque, sur le cours des choses. Il est vrai que l'intervention d'un acteur bien concret, les EtatsUnis, a brutalement introduit un tiers dans les interactions entre protagonistes en les faisant tous apparaître comme membres d'une communauté délinquante. Les limites de la méthode des ultimatums ne sont pas moins évidentes : ils peuvent faire percevoir la situation sous un angle nouveau mais ils ressemblent aussi beaucoup à l'immixtion d'un protagoniste supplémentaire. En tout état de cause, les Etats-Unis n'ont pas nécessairement de titre, autre que la relation de force, pour s'identifier à la Loi et, moins encore, pour le faire admettre par les Colombiens même si leurs lois sont défaillantes.

\section{NOTES}

1.Chiffre obtenu par l'addition des homicides annuels à partir des données officielles fournies par la police. Cf. Policia Nacional, Criminalidad 1995, Republica de Colombia, 1996, graphique $1 \mathrm{~F}$. Il faut sans doute tenir déduire de ce total le nombre d'homicides correspondant à une situation " normale " en Colombie. Même au cours des années 1960, le taux d'homicides est rarement descendu au dessous de 15 pour 100.000.

2.Cf. M. Uribe et T. Vasquez, Enterrar y callar, Bogota, Comite Permanente por la Defensa de los Derechos Humanos, 1996.

3.Selon les estimations, le nombre des morts de la UP varie entre 1500 et 3000 . 
4.Je renvoie à ce sujet aux livres de la Comision de estudios sobre la violencia, Colombia, Violencia y Democracia, Bogota, Universidad Nacional de Colombia 1987, de M. Deas et F. Gaitan Daza, Dos ensayos especulatios sobre la violencia en Colombia, Bogota, Departamento Nacional de Planeacion, 1995, aux deux volumes de la revue Controversia, $n^{\circ}$ 151-152 et 153-154 parus sous le titre Un Pais en construccion, en 1989, ainsi qu'à mon chapitre " Présent, passé, futur de la violence " in. J. M. Blanquer et C. Gros (dir.), La Colombie à l'aube du Troisième Millénaire, Paris, Editions de l'Institut des Hautes Etudes de l'Amérique Latine, 1996.

5.Sur la notion de " mise en intrigue ", Cf. P. Ricoeur, Temps et Récit, tome 1, Paris, Seuil, 1983.

6.Selon l'estimation présentée par les auteurs de l'étude citée plus haut Colombie, violencia Elle a par la suite souvent été répétée sans vérification approfondie.

7.Cf. D. Betancourt et M. Garcia, " Colombie: les mafias de la drogue ", Problèmes d'Amérique Latine, $n^{\circ} 18$, juin-sept 1995, P. 73-82.

8.Les émeraudes représentent selon les moments le deuxième ou troisième produit d'exportation. Depuis plusieurs siècles, c'est l'un des pôles chroniques de violence. Nombre des grands bandits les plus fameux en sont issus. A l'heure actuelle, les mines sont attribuées par contrat public à des compagnies, mais une grande partie des exportations est clandestine. Le mixte de légalité et d'illégalité confère à cette zone, proche de Bogota mais en même temps isolée, une fonction privilégiée dans les stratégies de violence. Gonzalo Rodriguez Gacha, l'associé de Pablo Escobar, en provenait. Une guerre entre deux camps rivaux pour l'hégémonie sur la zone a sévi à la fin des années 1980, faisant plusieurs milliers de morts.

9.Cf. C. Echandia, " Colombie : dimensions économiques de la violence et de la criminalité ", Problèmes d'Amérique Latine, n¹6, janv-mars 1995, p. 74.

10.Les FARC ont réussi à détruire les bandes paramilitaires implantées dans le Putumayo, département stratégique pour les activités liées à la drogue, mais les paramilitaires de Rodriguez Gacha ont éliminé dans d'autres régions, en particulier dans le Moyen Magdalena, nombre de collaborateurs ou d'alliés des FARC, à commencer par les cadres et militants de l'Union Patriotique.

11.Sans doute n'est-ce pas un hasard si nombre d'embuscades les plus meurtrières des guérillas contre les militaires se sont déroulées dans le Putumayo et le Caqueta, épicentres avec le Guaviare de l'économie de la cocaïne.

12.Les guérillas depuis quelques années visent du reste aussi à s'assurer la haute main sur les investissements locaux en mettant en tutelle les maires, quelle que soit l'obédience politique de ceux-ci.

13.Cf. l'analyse en termes de stratégie militaire présentée par Alfredo Rangel Suarez dans " Colombia : la guerra irregular en el fin de siglo ", Analisis Politico, $\mathrm{n}^{\circ} 28$, mai-août 1996, p. 74-84.

14.Dans plusieurs régions sous hégémonie des guérillas, les listes qui se réclamaient des partis politiques directement placés sous leurs auspices recueillent de moins en moins de voix depuis dix ans. Imputer ce recul à la seule terreur reviendrait à ignorer la méfiance de l'électorat envers ces partis ambigus. Les guérillas tournent à présent la difficulté en donnant leur aval à des candidats des partis traditionnels qu'elles mettent sous surveillance une fois qu'ils sont élus.

15.Sur les rapports sociaux dans l'Urabá, cf. G. Martin, " Desarrollo economico, sindicalismo y proceso de paz en Urabá ", Universidad de los Andes, Tesis de Grado, 1986, F. Botero, Urabá: Colonizacion, Violencia y Crisis del Estado, Medellín, Ed. 
Universidad de Antioquia, 1991 et C.I. Carcia Urabá, Region, Actores y Conflicto 1960-1990, Bogota CEREC, 1996.

16.Cf. Francisco Thoumi, Economia, Politica y Narcotrafico, Bogota, Tercer Mundo, 1994.

17. Cette problématique est notamment soutenue par Edgar Reveiz dans son livre Democratizar para sobrevivir, Bogota, Poligrupo Comunicacion, 1989.

18. Comme l'a bien montré J. A. Bejarano dans son chapitre " Democracia, conflicto y eficencia economica " in J. A. Bejarano, Construir la Paz, Bogota, Presidencia de la Republica, 1990, p. 143-171.

19.The sicilian mafia. The business of private protection, Cambridge University Press, 1993.

20.Les lois de l'accumulation capitaliste s'imposent cependant même aux FARC. Dans certains départements, le Guaviare notamment, une grande propriété consacrée à la coca s'est peu à peu mise en place et représente désormais un pourcentage considérable de la production.

21.Cf. R. Catanzaro, " La mafia et les recherches sur la mafia en Italie ", Déviance et société, vol. 19, n², p. 201-213.

22.Parmi lesquels la destruction d'un avion d'Avianca en plein vol.

23. Comme cela a été le cas pour certains des principaux dirigeants du parti libéral après 1950 .

24.Toutes ces données sont reprises de deux études de M. Rubio, " Homicidios, justicia, mafia y capital social. Otro ensayo sobre la violencia colombiana ", Document du CEDE, Université des Andes, 1996 ; et " Capital social, educacion y delincuencia juenil en Colombia ", CEDE, Université des Andes, 1996.

25.Cf. Alejandro Garcia, Hijos de la violencia, Madrid, Los libros de la Catarata, 1996.

26.Par ailleurs ancien membre du cartel de Medellín avant de se transformer en adversaire numéro un de Pablo Escobar

27.Cf. Maria Victoria Uribe, Matar, Rematar y Contramatar, Las Masacres de la Violencia en el Tolima, Bogota, CINEP, 1992.

28.Ils ont décapité devant Gloria Cuartas et des élèves d'une école un tout jeune enfant. 29. Ce type d'antagonisme est au centre la réflexion d'E. Balibar dans le texte "Violence : idéalité et cruauté ", in F. Héritier (dir.), De la Violence, Paris, Odile Jacob, 1996, p. 55-88. Il s'agit pour lui de mettre en relation l'affirmation d'idéaux avec la violence. 30.Des leaders du cartel de Medellin, comme les frères Ochoa, n'ont été condamnés qu'à des peines de deux ans de prison. Un des responsables du "cartel du Nord du Valle du Cauca", soupçonné de multiples massacres collectifs, n'a été condamné qu'à une peine de trois ans d'emprisonnement, ensuite portée à six ans. Face à la mesure de " désertification " que les Etats-Unis ont infligée à la Colombie, le gouvernement et le Congrès ont à la fin de 1996 accru les peines et pris des mesures pour pouvoir exproprier les biens acquis par les trafiquants. Eu égard au système sophistiqué de dissimulation de ces biens mis au nom d'hommes de paille, l'on peut douter que cette dernière disposition ait des effets à court terme.

31.Certains auteurs comme F. Leal Buitrago (cf. El oficio de la guerra, La seguridad Nacional en Colombia, Bogota, Tercer Mundo, 1994) attribuent aux forces militaires colombiennes une presque complète " autonomie ". Outre que le terme est loin d'être clair, il faut distinguer entre la capacité d'imposer un projet de société au pouvoir civil (comme les militaires argentins ou brésiliens) et la simple autonomie opérationnelle. Considérés depuis toujours avec un mépris social par les élites sociales, prisonniers de 
la forte tradition civiliste, dotés d'une formation géopolitique limitée dans les écoles de guerre, soumis aux décisions du Congrès pour la fixation de leur budget, longtemps très réduit, les militaires n'ont guère pu s'affirmer dans le domaine politique. En contrepartie, les élites civiles leur ont lâché les rênes pour la conduite des opérations militaires. Mais c'est un cadeau empoisonné. Faute de dessein politique clair, les militaires agissent au jour le jour. La référence à la " sécurité nationale " est purement rhétorique. L'on ne voit pas de responsables militaires qui aient élaboré une théorie de cette sécurité.

32.Je renvoie sur ce point à mon ouvrage L'ordre et la Violence, Evolution sociopolitique de la Colombie entre 1930 et 1953, Paris, Editions de l'Ecole des Hautes Etudes en Sciences Sociales, 1987.

33.Cf. F-X. Guerra, Le Mexique, De l'Ancien Régime à la Révolution, 2 vol, Paris, L'harmattan, 1985 et M-D. Demélas, L'invention politique, Bolivie, Equateur, Pérou au XIXè siècle, Paris, ERC,1992.

34.Nous empruntons tous les éléments de la narration qui suit à l'excellent livre de Adolfo Leon Atehortua Cruz, El Poder y la Sangre, Les Historias de Trujillo, Valle, Cali, Gobernacion del alle del Cauca, 1996.

35.Sur ce thème, cf. P. Ricoeur, Soi-même comme autre, Paris, Seuil, 1990.

\section{RÉSUMÉS}

The difficulty to think events by giving them a meaning is manifest in Colombia. Though it once was thought under the sign of the 1940s Violencia prolongation, violence has today become common place. This does not occur without consequence to the individual torn apart between multiple tensions. Trapped between several networks, condemned to live from day to day, one still expects « help » from the State. "There is no synthesis between these multiple tensions » for Daniel Pécault. As a consequence, the only temporal constancy for the individual remains in « the story of a trajectory leading him from one situation to the other ». In the same way does public opinion lose all capacity to generate an emotion when faced to the multiplicity of the forms of violence. Violence therefore appears in Colombia as a mode of operation corroding the institutions themselves, without anyone or anything able to impose its will without being a new protagonist in a generalised war that is meant to last according to all expectation.

INDEX

Index géographique : Amérique du Sud, Colombie

Thèmes : FARC-EP

\section{AUTEUR}

DANIEL PÉCAUT

Directeur d'études à l'EHESS, CEMS 